Copyright (C) 2013 IEEE. Personal use of this material is permitted. Permission from IEEE must be obtained for all other uses, in any current or future media, including reprinting/republishing this material for advertising or promotional purposes, creating new collective works, for resale or redistribution to servers or lists, or reuse of any copyrighted component of this work in other works. 


\title{
The role of the generalised continuous algebraic Riccati equation in impulse-free continuous-time singular LQ optimal control
}

\author{
Augusto Ferrante and Lorenzo Ntogramatzidis
}

\begin{abstract}
In this paper the role that the continuous-time generalised Riccati equation plays within the context of singular linear-quadratic optimal control is analysed. To date, the importance of the continuous-time generalised Riccati equation in the context of optimal control has not been understood. This note addresses this point. We show in particular that when the continuous-time (constrained) generalised Riccati equation admits a symmetric solution, the corresponding linear-quadratic (LQ) problem admits an impulse-free optimal control.
\end{abstract}

\section{INTRODUCTION}

It is well known that the solution of the classic finite and infinite-horizon LQ optimal control problem strongly depends on the matrix weighting the input in the cost function, traditionally denoted by $R$. When $R$ is positive definite, the problem is said to be regular (see e.g. [1], [11]), whereas when $R$ is positive semidefinite, the problem is called singular. The singular cases have been treated within the framework of geometric control theory, see for example [9], [18], [15], [13] and the references cited therein. In particular, in [9] and [18] it was proved that an optimal solution of the singular LQ problem exists for all initial conditions if the class of allowable controls is extended to include distributions.

The so-called continuous-time generalised Riccati equation was defined in the continuous time by following the analogy with the discrete case, in such a way that the inverse of $R$ appearing in the standard Riccati equation is replaced by its pseudo-inverse. Some conditions under which this equation admits a stabilising solution were investigated in terms of the so-called deflating subspaces of the extended Hamiltonian pencil. Some preliminary work on the continuous-time algebraic Riccati equation within the context of spectral factorisation has been carried out in [2] and [17]. Nevertheless, to date the role of this equation in relation to the solution of optimal control problems in the continuous time has not been fully explained. The goal of this paper is to fill this gap, by providing a counterpart of the results in [6] for the continuous case. In particular, we describe the role that the generalised continuous algebraic

Partially supported by the University of Padova under grant $n$. CPDA111238 "A Unifying Framework for Spectral Estimation and Matrix Completion: A New Paradigm for Identification, Estimation, and Signal Processing" and by the Australian Research Council under the grant FT120100604

A. Ferrante is with Dipartimento di Ingegneria dell'Informazione, Università di Padova, via Gradenigo, 6/B - 35131 Padova, Italy. augusto@dei.unipd.it

L. Ntogramatzidis is with the Department of Mathematics and Statistics, Curtin University, Perth (WA), Australia. L.Ntogramatzidis@curtin.edu.au
Riccati equation plays in singular LQ optimal control. Such role does not trivially follow from the analogy with the discrete case. Indeed, in the continuous time, whenever the optimal control involves distributions, none of the solutions of the generalised Riccati equation is optimising. The goal of this paper is to explain the connection of the generalised continuous-time algebraic Riccati equation and of the generalised Riccati differential equation - which is also defined by substitution of the inverse of $R$ with the pseudo-inverse - and the solution of the standard LQ optimal control problem with infinite and finite horizons, respectively. We will show that when the generalised Riccati equation possesses a symmetric solution, both the finite and the infinite-horizon LQ problems admit an impulse-free solution. Moreover, such control can always be expressed as a state-feedback, where the gain can be obtained from the solution of the generalised continuoustime algebraic/differential Riccati equation.

This is the conference version of a longer journal paper submitted by the same authors to Automatica.

\section{GENERALISED RICCATI EQUATIONS AND SINGULAR LQ PROBLEMS}

Consider the standard linear time-invariant state differential equation

$$
\dot{x}(t)=A x(t)+B u(t),
$$

with the constraint on the initial state $x(0)=x_{0} \in \mathbb{R}^{n}$. Consider the matrices $Q \in \mathbb{R}^{n \times n}, S \in \mathbb{R}^{n \times m}$, and $R \in \mathbb{R}^{m \times m}$. We denote by $\Pi$ the Popov matrix

$$
\Pi \triangleq\left[\begin{array}{cc}
Q & S \\
S^{\mathrm{T}} & R
\end{array}\right],
$$

which we assume to be symmetric and positive semidefinite. We do not assume that $R$ is invertible.

The standard finite-horizon LQ problem consists in the minimisation of the performance index

$$
\begin{gathered}
J_{T, H}\left(x_{0}, u\right)=\int_{0}^{T}\left[\begin{array}{cc}
x^{\mathrm{T}}(t) & u^{\mathrm{T}}(t)
\end{array}\right]\left[\begin{array}{cc}
Q & S \\
S^{\mathrm{T}} & R
\end{array}\right]\left[\begin{array}{l}
x(t) \\
u(t)
\end{array}\right] d t, \\
+x^{\mathrm{T}}(T) H x(T)
\end{gathered}
$$

where $T \in \mathbb{R}_{+}$and $H=H^{\mathrm{T}} \geq 0$.

In this paper we study the solutions of this optimisation problem in relation with the solution of the following differential matrix equation

$$
\begin{aligned}
& \dot{P}(t)+P(t) A+A^{\mathrm{T}} P(t) \\
&-(S+P(t) B) R^{\dagger}\left(S^{\mathrm{T}}+B^{\mathrm{T}} P(t)\right)+Q=0,
\end{aligned}
$$


which will be referred to as the generalised Riccati differential equation $\operatorname{GRDE}(\Sigma)$. This equation generalises the standard Riccati differential equation to the case in which $R$ is possibly singular. In this paper, we also consider the so-called infinite-horizon LQ problem, which consists in the minimisation of the performance index

$$
J_{\infty}\left(x_{0}, u\right)=\int_{0}^{\infty}\left[\begin{array}{ll}
x^{\mathrm{T}}(t) & u^{\mathrm{T}}(t)
\end{array}\right]\left[\begin{array}{cc}
Q & S \\
S^{\mathrm{T}} & R
\end{array}\right]\left[\begin{array}{l}
x(t) \\
u(t)
\end{array}\right] d t .
$$

To this end, we will provide a characterisation of the solutions of the algebraic equation

$$
X A+A^{\mathrm{T}} X-(S+X B) R^{\dagger}\left(S^{\mathrm{T}}+B^{\mathrm{T}} X\right)+Q=0,
$$

which is referred to as the generalised continuous algebraic Riccati equation $\operatorname{GCARE}(\Sigma)$. In this equation, the symbol $\dagger$ denotes the Moore-Penrose matrix pseudo-inversion. This equation represents a generalisation of the classic continuous algebraic Riccati equation arising in infinite-horizon LQ problems since here $R$ is allowed to be singular. Eq. (6), along with the condition

$$
\operatorname{ker} R \subseteq \operatorname{ker}(S+X B),
$$

where the symbol $\operatorname{ker} M$ denotes the null-space of a matrix $M$, is referred to as constrained generalised continuous algebraic Riccati equation, and is denoted by $\operatorname{CGCARE}(\Sigma)$. Observe that from (2) we have $\operatorname{ker} R \subseteq \operatorname{ker} S$, which implies that (7) is equivalent to $\operatorname{ker} R \subseteq \operatorname{ker}(X B)$.

Let $G \triangleq I_{m}-R^{\dagger} R$. Hence, $\operatorname{ker} R=\operatorname{im} G$, where the symbol $\operatorname{im} G$ stands for the image (or range) of $G$. Moreover, we consider a non-singular matrix $T=\left[T_{1} \mid T_{2}\right]$ where $\operatorname{im} T_{1}=$ $\operatorname{im} R$ and $\operatorname{im} T_{2}=\operatorname{im} G$, and we define $B_{1} \triangleq B T_{1}$ and $B_{2} \triangleq B T_{2}$. Finally, to any $X=X^{\mathrm{T}} \in \mathbb{R}^{n \times n}$ we associate the matrices

$$
\begin{aligned}
Q_{X} & \triangleq Q+A^{\mathrm{T}} X+X A, \\
S_{X} & \triangleq S+X B, \\
K_{X} & \triangleq R^{\dagger}\left(S^{\mathrm{T}}+B^{\mathrm{T}} X\right)=R^{\dagger} S_{X}^{\mathrm{T}}, \\
A_{X} & \triangleq A-B K_{X}, \\
\Pi_{X} & \triangleq\left[\begin{array}{cc}
Q_{X} & S_{X} \\
S_{X}^{\mathrm{T}} & R
\end{array}\right] .
\end{aligned}
$$

When $X$ is a solution of $\operatorname{CGCARE}(\Sigma)$, then $K_{X}$ is the corresponding gain matrix, $A_{X}$ the associated closed-loop matrix, and $\Pi_{X}$ is the so-called dissipation matrix.

Remark 2.1: We begin by observing that an important difference between the continuous and the discrete-time generalised Riccati equations is the fact that in the continuous case, differently from the discrete case [6], it is not true that all symmetric and positive semidefinite solutions of $\operatorname{GCARE}(\Sigma)$ are also solutions of $\operatorname{CGCARE}(\Sigma)$. Consider for example the following example, where

$$
\begin{aligned}
& A=\left[\begin{array}{cc}
-8 & 0 \\
0 & -4
\end{array}\right], \quad B=\left[\begin{array}{ll}
6 & 0 \\
0 & 3
\end{array}\right], \\
& Q=\left[\begin{array}{cc}
16 & 0 \\
0 & 0
\end{array}\right], \quad S=\left[\begin{array}{ll}
0 & 0 \\
0 & 0
\end{array}\right], \quad R=\left[\begin{array}{cc}
0 & 0 \\
0 & 25
\end{array}\right] .
\end{aligned}
$$

It is a matter of direct substitution to verify that $X=\left[\begin{array}{ll}1 & 0 \\ 0 & 0\end{array}\right]$ is a solution of $\operatorname{GCARE}(\Sigma)$. However, one immediately verifies that $\operatorname{ker} R$ is spanned by the vector $\left[\begin{array}{l}1 \\ 0\end{array}\right]$ whereas $\operatorname{ker}(S+X B)$ is spanned by $\left[\begin{array}{l}0 \\ 1\end{array}\right]$, so that (7) is not satisfied.

The following simple result holds.

Lemma 2.1: Let $X$ be a solution of $\operatorname{CGCARE}(\Sigma)$. Then, $X B_{2}=0$.

Proof: From (7) and $\operatorname{ker} R=\operatorname{im} G$, it is found that $(S+$ $X B) G=0$. Moreover, since $\Pi$ is positive semidefinite, we have $\operatorname{ker} S \supseteq \operatorname{ker} R$. This means that there exists $K \in \mathbb{R}^{n \times m}$ such that $S=K R$. Therefore, $S R^{\dagger} R=K R R^{\dagger}=K R=S$, and $S G=S-S R^{\dagger} R=0$. Hence, $X B_{2}=0$.

Lemma 2.2: Let $\tilde{A}=A-B R^{\dagger} S^{\mathrm{T}}$ and $\tilde{Q}=Q-S R^{\dagger} S^{\mathrm{T}}$. Then, $\tilde{Q} \geq 0$ and $\operatorname{GCARE}(\Sigma)$ defined in (6) has the same set of symmetric solutions of the following equation:

$$
X \tilde{A}+\tilde{A}^{\mathrm{T}} X-X B R^{\dagger} B^{\mathrm{T}} X+\tilde{Q}=0 .
$$

Proof: Since $\tilde{Q}$ is the generalised Schur complement of $R$ in $\Pi, \tilde{Q}$ is positive semidefinite because such is also $\Pi$. The rest of the proof is a matter of verifying that (6) is obtained by substitutions of $\tilde{A}$ and $\tilde{Q}$ into (13).

Remark 2.2: The result established for $\operatorname{GCARE}(\Sigma)$ in Lemma 2.2 extends without difficulties to the generalised Riccati differential equation $\operatorname{GRDE}(\Sigma)$. Indeed, we easily see that (4) has the same set of symmetric solutions of the equation:

$$
\dot{P}(t)+P(t) \tilde{A}+\tilde{A}^{\mathrm{T}} P(t)-P(t) B R^{\dagger} B^{\mathrm{T}} P(t)+\tilde{Q}=0 .
$$

Lemma 2.3: Let $X=X^{\mathrm{T}}$ be a solution of $\operatorname{CGCARE}(\Sigma)$. Let $\mathscr{R}\left(\tilde{A}, B_{2}\right)$ be the reachable subspace of the pair $\left(\tilde{A}, B_{2}\right)$. The following three facts hold true:

(i) $\operatorname{ker} X \subseteq \operatorname{ker} \tilde{Q}$;

(ii) $X \mathscr{R}\left(\tilde{A}, B_{2}\right)=\{0\}$;

(iii) $\tilde{Q} \mathscr{R}\left(\tilde{A}, B_{2}\right)=\{0\}$.

Proof: (i). Let $\xi \in \operatorname{ker} X$. From (13) we get $\xi^{\mathrm{T}} \tilde{Q} \xi=0$. Since $\tilde{Q} \geq 0$, we get $\Lambda \xi=0$. Hence, $\operatorname{ker} X \subseteq \operatorname{ker} \tilde{Q}$.

(ii). Let $\xi \in \operatorname{ker} X$. From (13) we find $X \tilde{\tilde{A}} \xi=0$., which implies that $\operatorname{ker} X$ is $\tilde{A}$-invariant. Invoking Lemma 2.2, we see that the subspace $\operatorname{ker} X$ contains $\operatorname{im} B_{2}$. Hence, it contains $\mathscr{R}\left(\tilde{A}, B_{2}\right)$ that is the smallest $\tilde{A}$-invariant subspace containing $\operatorname{im} B_{2}$. This implies $\mathscr{R}\left(\tilde{A}, B_{2}\right) \subseteq \operatorname{ker} X$.

(iii). This follows directly from the chain of inclusions $\mathscr{R}\left(\tilde{A}, B_{2}\right) \subseteq \operatorname{ker} X \subseteq \operatorname{ker} \tilde{Q}$. 


\section{THE FINITE-HORIZON LQ PROBLEM}

In this section, our attention is focussed on the finitehorizon LQ problem as defined in Section II.

Lemma 3.1: Let $H=H^{\mathrm{T}} \geq 0$ be such that $H \mathscr{R}\left(\tilde{A}, B_{2}\right)=$ $\{0\}$. If CGCARE $(\Sigma)$ (6-7) admits solutions, the generalised Riccati differential equation

$$
\begin{aligned}
& \dot{P}_{T}(t)+P_{T}(t) A+A^{\mathrm{T}} P_{T}(t) \\
& \quad-\left(S+P_{T}(t) B\right) R^{\dagger}\left(S^{\mathrm{T}}+B^{\mathrm{T}} P_{T}(t)\right)+Q=0,
\end{aligned}
$$

with the terminal condition

$$
P_{T}(T)=H
$$

admits a unique solution for all $t \leq T$, and this solution satisfies $P_{T}(t) B G=0$ for all $t \leq T$.

Proof: Consider a set of coordinates in the input space such that the first coordinates span $\operatorname{im} R$ and the second set of coordinates spans $\operatorname{im} G=\operatorname{ker} R$. In this basis $R$ can be written as $R=\left[\begin{array}{rr}R_{1} & 0 \\ 0 & 0\end{array}\right]$ with $R_{1}$ being invertible. In the same basis, matrix $B$ can be partitioned accordingly as $B=\left[\begin{array}{ll}B_{1} & B_{2}\end{array}\right]$ as shown above. Consider the change of basis matrix $U=$ $\left[\begin{array}{ll}U_{1} & U_{2}\end{array}\right]$ where $\operatorname{im} U_{1}=\mathscr{R}\left(\tilde{A}, B_{2}\right)$, so that

$U^{-1} \tilde{A} U=\left[\begin{array}{cc}\tilde{A}_{11} & \tilde{A}_{12} \\ O & \tilde{A}_{22}\end{array}\right], \quad U^{-1} B_{1}=\left[\begin{array}{l}B_{11} \\ B_{12}\end{array}\right], \quad U^{-1} B_{2}=\left[\begin{array}{c}B_{21} \\ O\end{array}\right]$ and $U^{\mathrm{T}} \tilde{Q} U=\left[\begin{array}{cc}O & O \\ O & \tilde{Q}_{22}\end{array}\right]$ where we have used the fact that $\tilde{Q} \mathscr{R}\left(\tilde{A}, B_{2}\right)=\{0\}$. Since we are assuming $H \mathscr{R}\left(\tilde{A}, B_{2}\right)=\{0\}$, we can write $U^{\mathrm{T}} H U=\left[\begin{array}{cc}O & O \\ O & H_{22}\end{array}\right]$. Consider the matrix function $P_{T}(t)=\left[\begin{array}{ll}O & O \\ O & P_{22}(t)\end{array}\right]$, where $P_{22}(t)$ satisfies

$$
\begin{aligned}
& \dot{P}_{22}(t)+P_{22}(t) \tilde{A}_{22}+\tilde{A}_{22}^{\mathrm{T}} P_{22}(t)-P_{22}(t) V P_{22}(t)+\tilde{Q}_{22}=0 \\
& P_{22}(T)=H_{22},
\end{aligned}
$$

in which $V$ is the sub-block 22 of the matrix $B R^{\dagger} B^{\mathrm{T}}$. Since $\Pi=\Pi^{\mathrm{T}} \geq 0$ and $H=H^{\mathrm{T}} \geq 0$, from [8, Corollary 2.4] we conclude that both (15) and (17) admit a unique solution defined in $(-\infty, T]$. It is easy to see that $P_{T}(t)=\left[\begin{array}{cc}O & O \\ O & P_{22}(t)\end{array}\right]$, where $P_{22}(t), t \in(-\infty, T]$, is the solution of (17-18), solves (15) and (16). We can therefore conclude that $P_{T}(t)$ is the unique solution of (15-16). Moreover, this solution satisfies $P_{T}(t) B_{2}=0$ for all $t \leq T$ since in the chosen basis $P_{T}(t) B_{2}=$ $\left[\begin{array}{cc}O & O \\ O & P_{22}(t)\end{array}\right]\left[\begin{array}{c}B_{21} \\ O\end{array}\right]=0$.

The following theorem is the first main result of this paper. It shows that when $\operatorname{CGCARE}(\Sigma)$ admit a solution, the finite-horizon LQ problem always admits an impulse-free solution. The proof is omitted.

Theorem 3.1: Let $\operatorname{CGCARE}(\Sigma)$ admit a solution. The finite-horizon LQ problem (3-1) admits impulse-free optimal solutions. All such solutions are given by

$$
u(t)=-R^{\dagger}\left(S^{\mathrm{T}}+B^{\mathrm{T}} P_{T}(t)\right) x(t)+G v(t),
$$

where $v(t)$ is an arbitrary regular function, and $P_{T}(t)$ is the solution of (15) with the terminal condition (16). The optimal cost is $x_{0}^{\mathrm{T}} P_{T}(0) x_{0}$.

\section{THE INFINITE-HORIZON LQ PROBLEM}

We are now interested in studying $P_{T}(0)$ when the terminal condition vanishes, i.e., when $H=0$, and the time interval increases. To this end, we consider a generalised Riccati differential equation where the time is reversed, and where the terminal condition becomes an initial condition, which is now equal to zero. More specifically, we consider the new matrix function $X(t)=P_{t}(0)=P_{T}(T-t)$. We re-write $\operatorname{GRDE}(\Sigma)$ as a differential equation to be solved forward:

$$
\begin{aligned}
& \dot{X}(t)= X(t) A+A^{\mathrm{T}} X(t) \\
& \quad-(S+X(t) B) R^{\dagger}\left(S^{\mathrm{T}}+B^{\mathrm{T}} X(t)\right)+Q, \\
& X(0)=0 .
\end{aligned}
$$

In the following theorem, the second main result of this paper is introduced. This theorem determines when the infinite-horizon LQ problem admits an impulse-free solution, and the set of optimal controls minimising the infinitehorizon cost $J_{\infty}\left(x_{0}, u\right)$ defined in (5) subject to the constraint (1).

Theorem 4.1: Suppose $\operatorname{CGCARE}(\Sigma)$ admits at least a symmetric solution, and that for every $x_{0}$ there exists an input $u(t) \in \mathbb{R}^{m}$, with $t \geq 0$, such that $J_{\infty}\left(x_{0}, u\right)$ in (5) is finite. Then:

(1) A solution $\bar{X}=\bar{X}^{\mathrm{T}} \geq 0$ of $\operatorname{CGCARE}(\Sigma)$ is obtained as the limit of the time varying matrix generated by integrating (20) with the zero initial condition (21).

(2) The value of the optimal cost is $x_{0}^{T} \bar{X} x_{0}$.

(3) $\bar{X}$ is the minimum positive semidefinite solution of $\operatorname{CGCARE}(\Sigma)$.

(4) The set of all optimal controls minimising $J_{\infty}$ in (5) can be parameterised as

$$
u(t)=-R^{\dagger} S_{\bar{X}}^{\mathrm{T}} x(t)+G v(t),
$$

with arbitrary $v(t)$.

The proof of this result can be carried out along the same lines of the proof of Theorem 2.1 in [6], and is omitted.

\section{A. Infinite-horizon $L Q$ problem and stabilisability}

In this section we introduce some concepts that will shed light into the infinite-horizon LQ problem with closed-loop stability. Most of these concepts are adaptation of several results that were presented in [6] to the continuous time. First, since as aforementioned the Popov matrix $\Pi$ is assumed symmetric and positive semidefinite, we can consider a factorisation of the form

$$
\Pi=\left[\begin{array}{cc}
Q & S \\
S^{\mathrm{T}} & R
\end{array}\right]=\left[\begin{array}{c}
C^{\mathrm{T}} \\
D^{\mathrm{T}}
\end{array}\right]\left[\begin{array}{ll}
C & D
\end{array}\right],
$$

where $Q=C^{\mathrm{T}} C, S=C^{\mathrm{T}} D$ and $R=D^{\mathrm{T}} D$. The following results hold:

- Let $X$ be a solution of $\operatorname{GCARE}(\Sigma)$. Then, $\operatorname{ker} X$ is an output-nulling subspace of the quadruple $(A, B, C, D)$ and $-K_{X}$ is a friend of $\operatorname{ker} X$. 
- Let $X=X^{\mathrm{T}}$ be a solution of $\operatorname{CGCARE}(\Sigma), C_{X} \triangleq C-$ $D R^{\dagger} S^{\mathrm{T}}$ and

$$
\mathscr{R}_{0, X} \triangleq \operatorname{im}\left[\begin{array}{llll}
B_{2} & A_{X} B_{2} & \ldots & A_{X}^{n-1} B_{2}
\end{array}\right] .
$$

Then, (i) $\mathscr{R}_{0, X} \subseteq \operatorname{ker} C_{X}$; (ii) $X \mathscr{R}_{0, X}=\{0\}$; (iii) $\mathscr{R}_{0, X}$ coincides with the largest reachability subspace on the output nulling subspace $\operatorname{ker} X$, i.e.,

$$
\mathscr{R}_{0, X}=\left\langle A_{X}, \operatorname{ker} X \cap B \operatorname{ker} D\right\rangle .
$$

- $\mathscr{R}_{0, X}$ is independent of the solution $X=X^{\mathrm{T}}$ of CGCARE $(\Sigma)$. Moreover, $A_{X}$ restricted to this subspace does not depend on the particular solution $X=X^{\mathrm{T}}$ of $\operatorname{CGCARE}(\Sigma)$, i.e.,

$$
\mathscr{R}_{0, X}=\mathscr{R}_{0, Y} \quad \text { and }\left.\quad A_{X}\right|_{\mathscr{R}_{0, X}}=\left.A_{Y}\right|_{\mathscr{R}_{0, Y}},
$$

where $X$ and $Y$ are two symmetric solutions of $\operatorname{CGCARE}(\Sigma)$ while $A_{X}$ and $A_{Y}$ are the corresponding closed-loop matrices.

The proofs of these results follow by adapting Theorem 4.1, Lemma 4.1, Theorem 4.2 and Theorem 4.3 in [6] to the continuous time generalised Riccati equation.

From these considerations, it turns out that the eigenvalues of the closed-loop matrix $A_{X}$ restricted to the subspace $\mathscr{R}_{0, X}$ are independent of the particular solution $X=X^{\mathrm{T}}$ of $\operatorname{CGCARE}(\Sigma)$ considered. This means that these eigenvalues are present in the closed-loop regardless of the solution $X=X^{\mathrm{T}}$ of $\operatorname{CGCARE}(\Sigma)$ that we consider. On the other hand, we have also observed that $\mathscr{R}_{0, X}$ coincides with the smallest $A_{X}$-invariant subspace containing $\operatorname{ker} X \cap B \operatorname{ker} D$. It follows that it is always possible to find a matrix $L$ that assigns all the eigenvalues of the map $\left(A_{X}+B_{2} L\right)$ restricted to the reachable subspace $\mathscr{R}_{X, 0}$, by adding a further term $B_{2} L x(t)$ to the feedback control law, because this does not change the value of the cost with respect to the one obtained by $u(t)=-K_{X} x(t)$. Indeed, the additional term only affects the part of the trajectory on $\mathscr{R}_{0, X}$ which is output-nulling. However, in doing so it may stabilise the closed-loop if $\operatorname{ker} X$ is externally stabilised by $-K_{X}$. Indeed, since $\mathscr{R}_{0, X}$ is output-nulling with respect to the quadruple $(A, B, C, D)$, it is also output-nulling for the quadruple $\left(A_{X}, B, C_{X}, D\right)$, and two matrices $\Xi$ and $\Omega$ exist such that

$$
\left[\begin{array}{l}
A_{X} \\
C_{X}
\end{array}\right] R_{0, X}=\left[\begin{array}{c}
R_{0, X} \\
0
\end{array}\right] \Xi+\left[\begin{array}{l}
B \\
D
\end{array}\right] \Omega,
$$

where $R_{0, X}$ is a basis matrix of $\mathscr{R}_{0, X}$. In order to find a matrix stabilise the system, we solve the former in $\Xi$ and $\Omega$, so as to find $L$ such that

$$
\left[\begin{array}{l}
A_{X}+B L \\
C_{X}+D L
\end{array}\right] R_{0}=\left[\begin{array}{c}
R_{0} \\
0
\end{array}\right] \Xi
$$

where the eigenvalues of $\Xi$ are the eigenvalues of the map $A_{X}+B L$ restricted to $\mathscr{R}_{0, X}$. Using the standard procedure of geometric control theory [16], we first compute the set of solutions of (25) in $\Xi$ and $\Omega$, which is given by

$$
\left[\begin{array}{l}
\Xi \\
\Omega
\end{array}\right]=\left[\begin{array}{c}
\hat{\Xi} \\
\hat{\Omega}
\end{array}\right]+\left[\begin{array}{l}
H_{1} \\
H_{2}
\end{array}\right] K
$$

for an arbitrary matrix $K$, where

$$
\left[\begin{array}{c}
\hat{\Xi} \\
\hat{\Omega}
\end{array}\right]=\left[\begin{array}{cc}
R_{0, X} & B \\
O & D
\end{array}\right]^{\dagger}\left[\begin{array}{l}
A_{X} \\
C_{X}
\end{array}\right] R_{0, X}
$$

and $\left[\begin{array}{l}H_{1} \\ H_{2}\end{array}\right]$ is a basis matrix of $\operatorname{ker}\left[\begin{array}{rr}R_{0, X} & B \\ O & D\end{array}\right]$. Since $\mathscr{R}_{0, X}$ is a reachability output-nulling subspace, it turns out that the pair $\left(\hat{\Xi}, H_{1}\right)$ is reachable. This implies that a matrix $K$ in (26) can always be found so that the eigenvalues of $\Xi$ are freely assignable (provided they come in complex conjugate pairs). Hence, we use such $K$ in (26) and then we compute $L=$ $-\Omega R_{0, X}^{\dagger}$. This choice guarantees that only the eigenvalues of $A_{X}$ restricted to $\mathscr{R}_{0, X}$ get affected by the use of $L$. From these considerations, it emerges that, given a symmetric solution $X$ of $\operatorname{CGCARE}(\Sigma)$, the infinite-horizon problem admits a stabilising solution if and only if the eigenvalues induced by the closed-loop matrix $A_{X}$ on the quotient space $\mathbb{R}^{n} / \mathscr{R}_{0, X}$ are all asymptotically stable.

\section{CONCLUDING REMARKS}

In this paper we established a new theory that showed that, when the $\operatorname{CGCARE}(\Sigma)$ admits solutions, the corresponding singular LQ problem admits an impulse-free solution, and the optimal control can be expressed in terms of a state feedback. A very interesting question, which is currently being investigated by the authors, is the converse implication of this statement: when the singular LQ problem admits a regular solution for all initial states $x_{0} \in \mathbb{R}^{n}$, does the $\operatorname{CGCARE}(\Sigma)$ admit at least one symmetric positive semidefinite solution? At this stage we can only conjecture that this is the case, on the basis of some preliminary work, but the issue is indeed an open and interesting one.

In the last part of the paper, we showed that a subspace can be identified that is independent of the particular solution of CGCARE considered, and that the closed-loop matrix restricted to this subspace does not depend on the particular solution of CGCARE. If such subspace is not zero, in the optimal control a further term can be added to the statefeedback generated from the solution of the Riccati equation that does not modify the value of the cost. This term can in turn be expressed in state-feedback form, and acts as a degree of freedom that can be employed to stabilise the closed-loop even in cases in which no stabilising solutions exists of the Riccati equation.

Future investigations will also focus on examining how the use of generalised Riccati equations in the continuous time can be used to parameterise the trajectories that solve the Hamiltonian differential equation, to the end of addressing LQ problems with constraints at the end-points and biased performance indexes along the lines of [3], [12], [4], [5].

\section{REFERENCES}

[1] B.D.O. Anderson and J.B. Moore. Optimal Control: Linear Quadratic Methods. Prentice Hall International, London, 1989.

[2] T. Chen and B.A. Francis. Spectral and inner-outer factorisations of rational matrices. SIAM Journal of Matrix Analysis and Applications, 10(1):1-17, 1989.

[3] A. Ferrante, G. Marro, and L. Ntogramatzidis, "A parametrization of the solutions of the finite-horizon LQ problem with general cost and boundary conditions”. Automatica, 41(8): 1359-1366, 2005. 
[4] A. Ferrante, and L. Ntogramatzidis, "A Unified Approach to the FiniteHorizon Linear Quadratic Optimal Control Problem”. European Journal of Control, 13/5: 473-488, 2007.

[5] L. Ntogramatzidis, and A. Ferrante, "On the solution of the Riccati differential equation arising from the LQ optimal control problem". Systems \& Control Letters, 59:114-121, 2010.

[6] A. Ferrante, L. Ntogramatzidis, The generalised discrete algebraic Riccati equation in LQ optimal control. Automatica, 49(2):471-478, 2013.

[7] A. Ferrante, L. Ntogramatzidis, The extended symplectic pencil and the finite-horizon LQ problem with two-sided boundary conditions. IEEE Transactions on Automatic Control, 58(8), 2102-2107, 2013.

[8] G. Freiling, G. Jank, and A. Sarychev. Non-blow-up conditions for Riccati-type matrix differential and difference equations. Results of Mathematics, 37:84-103, 2000.

[9] M.L.J. Hautus and L.M. Silverman. System structure and singular control. Linear Algebra and Its Applications, 50:369-402, 1983.

[10] V. Ionescu and C. Oară. Generalized continuous-time Riccati theory. Linear Algebra and Its Applications, 232:111-130, 1996.

[11] F.L. Lewis and V. Syrmos. Optimal Control. John Wiley \& Sons, New York, 1995.

[12] L. Ntogramatzidis, and G. Marro, "A parametrization of the solutions of the Hamiltonian system for stabilizable pairs". International Journal of Control, 78(7): 530-533, May 2005.

[13] D. Prattichizzo, L. Ntogramatzidis, and G. Marro, "A new approach to the cheap LQ regulator exploiting the geometric properties of the Hamiltonian system". Automatica, 44: 2834-2839, 2008.

[14] D. Rappaport and L.M. Silverman. Structure and stability of discretetime optimal systems. IEEE Transactions on Automatic Control, AC16:227-233, 1971.

[15] A. Saberi and P. Sannuti. Cheap and singular controls for linear quadratic regulators. IEEE Transactions on Automatic Control, AC32(3):208-219, March 1987.

[16] H.L. Trentelman, A.A. Stoorvogel, and M. Hautus. Control theory for linear systems. Springer, 2001.

[17] M. Weiss, "Spectral and inner-outer factorisations through the constrained Riccati equation". IEEE Transactions on Automatic Control, AC-39(3):677-681, 1994.

[18] J.C. Willems, A. Kìtapçi, and L.M. Silverman. "Singular optimal control: a geometric approach". SIAM Journal of Control and Optimization, 24(2):323-337, March 1986. 